\title{
PS Techniques and Surface Deformation Monitoring Application
}

\author{
Shaochun Dong Hongwei Yin Lulu Huang \\ School of Earth Sciences and Engineering \\ Nanjing University \\ Nanjing, China \\ dsc@nju.edu.cn
}

\author{
Chong $\mathrm{Xu}$ Yongchun Chen \\ Institute of Huainan Coal Technology and Engineering \\ Huainan, China
}

\begin{abstract}
Persistent Scatter (PS) InSAR is an extension to the conventional InSAR techniques. It offers a practical way to reduce the temporal and geometrical decorrelation, and atmospheric artifacts in conventional D-InSAR processing method, therefore can be successfully applied in long-time subtle surface deformation monitoring with multiple SAR images. Surface subsidence mapping due to underground coal mining is one of the application cases in this field. This paper firstly overviews the limitation of conventional D-InSAR technique and the background of PS technique, and then addresses the data processing of PS technique. Several PS related data processors are discussed with aspects of PS pixel identification, algorithm and applicable areas. Undergoing research on monitoring land subsidence in Huainan coal mining areas with PS-InSAR technique is presented as an application example in the end of the paper.
\end{abstract}

Keywords-persistent scatterer，D-InSAR，StaMPS，DePSI, surface deformation

\section{INTRODUCTION}

Over the last two decades, D-InSAR provides users with the capability of mapping and monitoring subtle changes of the ground surface with a precision of the order of $1 \mathrm{~cm}$ or less. This technique has been successfully implied in the measuring deformation from active volcanism inflations, co- and postseismic motions, glacier movements and ground subsidence. It has numerous advantages over traditional methods for deformation measurements, including its precise accuracy, high spatial coverage, continuous monitoring capability over month or annually in all weather and all time with a comparatively lower cost. However, temporal decorrelation, geometric decorrelation and atmospheric inhomogeneities limit the accuracy and effect of D-InSAR, therefore render some of the images unusable for D-InSAR application $[1,2,3,4,5,6,7]$. To be specified, temporal decorrelation makes InSAR measurements unfeasible over vegetated areas or locations where backscattering characteristics of the surface change with time [3]. Geometric decorrelation limits the number of InSAR pairs of SAR images that are suitable for D-InSAR processing [1]. Atmospheric inhomogeneities cause extra phase fringes in interferograms and reduce the accuracy of InSAR measurements $[1,4]$.
In the late 1990s, Permanent Scatterer (PS) technique was introduced that deals with these problems in a practical and innovative way $[1,7,8]$. This technique address problems of decorrelation and atmospheric delay by discerning stably reflecting objects at a pixel or subpixel level in each interferogram among a stack of differential interferograms $[8,9]$. PS technique was first achieved as an algorithm by A. Ferretti, F. Rocca, and C. Prati of the Technical University of Milan and has been protected by a patent [8]. This technique offers a systematic processing strategy, capable of exploit all available SAR images, by creating a stack of differential interferograms that have a common master image. Instead of analyzing the phase in the spatial domain, the phase of isolated coherent points is analyzed as a function of time and space [8]. This technique is widely used to detect field surface deformation due to underground mining, massive exploitation of underground water, volcanism inflations etc.

\section{THEORETICAL BACKGROUND AND DATA PROCESSING OF PS-INSAR}

It was noticed that some radar targets maintain stable backscattering characteristics over long time intervals (e.g. months or years) and for wide look angle variations $[1,2,3,4,10,11]$. The phase information from these targets is coherent so that they can be thought of as temporally-stable and highly reflective ground features or phase stable point-wise radar targets [1]. The key concept behind PS technique is to identify those targets whose phase histories match an assumed model of how displacement varies with time $[4,10,11]$.

The input of the Persistent scatterer interferometric process is a stack of differential interferograms (normally more than 20 differential interferograms) coregistered to a unique master image. PS pixels are identified by analysis of amplitude stability, coherence stability and Signal to Clutter Ratio (SCR), etc in the series of differential interferograms [8].

The key processing steps (Fig. 1) of PS technique are the following $[1,3,4,6,8,10,11,12$,$] :$ 


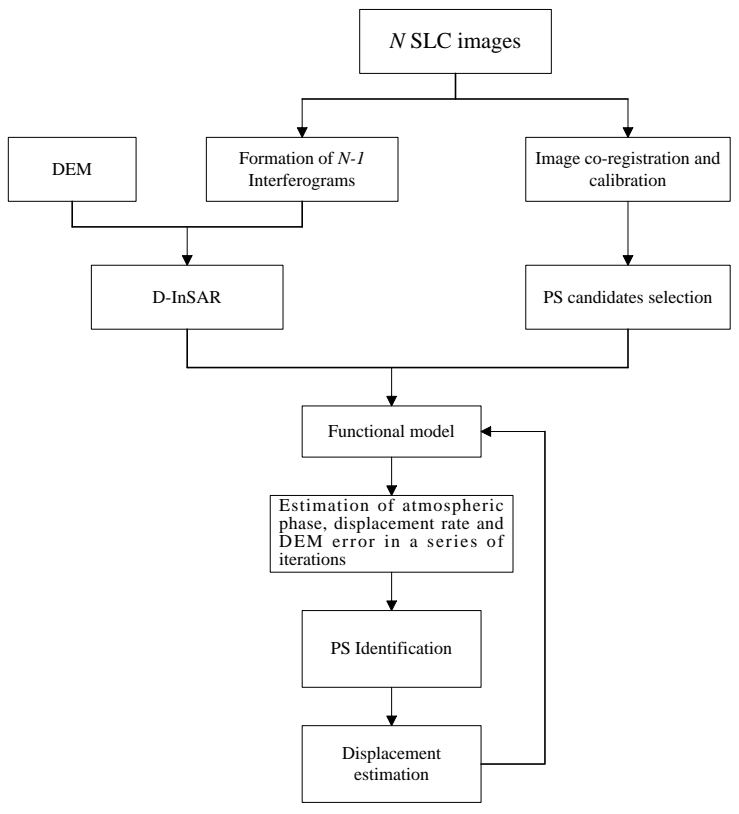

Fig. 1. Flowchart of PS method

\section{A. Computation of the Interferograms}

Given N SAR images (all images should be acquired from the same track), choose one master SLC image from those images. The master image should maximize the total coherence of the interferometric stack and minimize the sum decorrelation of all the interferograms, which depend on temporal baselines, perpendicular spatial baselines, mean Doppler centroid frequency difference and thermal noise. N-1 interferograms would be formed with respect to the same master image in this step.

\section{B. Differential Interferogram Formation}

Use DEM and precise orbit data to generate N-1 differential interferograms, and then eliminate the flat-Earth phase, calculate topographic phase and remove it from the interferograms. Hence, the wrapped phase in interferogram $\mathrm{i}$ can be expressed as:

$$
\phi_{i}=\phi_{\text {topo }, i}+\phi_{\text {defo }, i}+\phi_{\text {atm }, i}+\phi_{\text {noise }, i}
$$

Where $i$ refers to the ith interferogram in the stack of differential interferograms, $i \in[1, n-1] ; \phi_{\text {topo }, i}$ is the phase due to inaccuracy of the reference DEM; $\phi_{\text {defo }, i}$ represents the phase due to displacement of the pixel in the satellite line of sight (LOS) direction; $\phi_{a t m, i}$ represents the phase due to the atmospheric delays between repeated passes; $\phi_{\text {noise }, i}$ corresponds to the phase introduced by variable scattering background, thermal noise, co-registration errors, etc. The following steps are an iterative processing to estimate each of the phase components.

\section{PS Candidate Selections}

Estimate the PS probability for each pixel in the series of interferograms and coherence maps that been formed in the above steps. If a target shows coherence always greater than a suitable value, then this target would be selected as a PS candidate. Existing methods to identify of PS candidates could be subdivided into different groups that use the following parameterization respectively or combined: signal to clutter ratio; normalized amplitude dispersion; and supervised classification, etc. For example, points would be selected as PS candidates if the amplitude dispersion below a threshold.

\section{Preliminary Estimation}

An iteration process would be performed to get the preliminary estimation of atmospheric phase, displacement rate and DEM error at PS candidate positions in all interferograms. The estimations are performed between nearby points in order to limit atmospheric signal. The displacement is modeled using a linear combination of base functions.

\section{E. PS Selection}

Once the parameters at the points are computed, interpolate these estimates. Those most likely to be PS points with a threshold after converged on estimations would be added as additional PS pixels.

\section{F. Deformation Estimation}

Unwrapped the phase and other nuisance terms estimated at PS points and retrieve the phase due to the deformation.

\section{PS-INSAR PRPCESSORS}

Some algorithms are developed to fulfill the PS technique, including DePSI, StaMPS, IPTA, etc.

\section{A. DePSI}

Delft PS-InSAR processing package (DePSI) is a package for Persistent Scatter Interferometry developed by TU Delft, which simultaneously estimates the deformation for each PS. It adopts amplitude stability criterion to select PS pixels and use a temporal functional model for deformation estimation over time $[4,9,11]$. This method works best in urban areas where man-made structures increase the likelihood of finding a nonfluctuation scatterer in any given pixel [10]. For low SNR scatterers, the simple relationship between amplitude dispersion and phase stability breaks down, so that the method is no longer effective in rural areas [10].

\section{B. StaMPS}

Stanford Method for Persistent Scatters (StaMPS), adopts both amplitude and phase analysis to determine PS probability for individual pixels. This algorithm uses spatial phase stability, rather than a functional temporal model, to pick up PS pixels [4,9,10,11]. An initial selection of PS candidates is performed based only on amplitude analysis. And then the PS probability is refined by phase analysis through an iterative process. Once selected, the signal due to deformation in the PS pixels is isolated. It is applicable in areas undergoing non-steady deformation with no prior knowledge of the variations in deformation rate, so that it is effective in all terrains with or without buildings [10]. In other words, this algorithm can be also used effectively in rural areas. 


\section{GAMMA IPTA}

GAMMA is a remote sensing processor developed by a Swiss corporation. It provides a series of tools to conduct SAR and interferometric processing, advanced InSAR processing and analysis. IPTA (Interferometric Point Target Analysis) is one of GAMMA's modules, which constitutes a collection of tools to exploit the temporal and spatial characteristics of interferometric signatures collected from point targets to map surface deformation histories, terrain height and relative atmospheric path delay [13]. The analysis is achieved by an iterative improvement of the model parameters to optimally match the observed interferometric phases. The flowchart of IPTA is illustrated in Fig. 4.

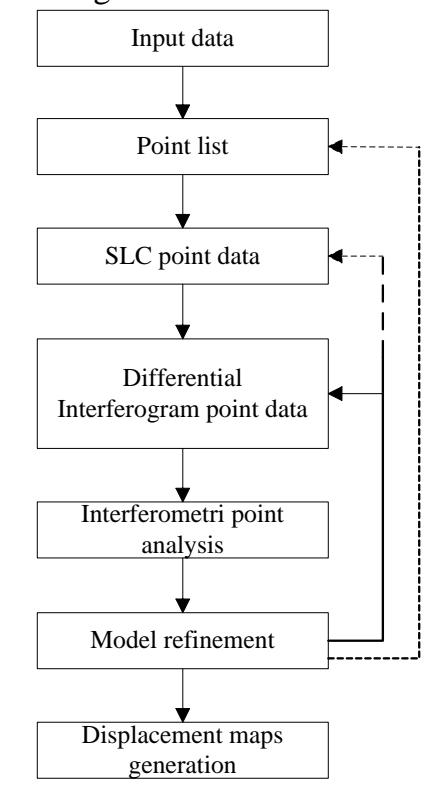

Fig. 2. Flowchart of IPTA method [13]

\section{APPLICATIONS}

Surface subsidence due to underground mining activity is a serious engineering, economic and environmental issue [14,15]. Over 8000 underground coal mines are operating in China and produce adverse environmental impacts such as loss of productive land, damage to underground facilities and above ground structures, decreasing stability of slopes and escarpments, contamination of ground water by acid drainage, and dewatering of streams and ground water supply.

For example, Huainan, in Anhui province, east China, is home to a number of active underground coal mines. The mines are mostly located in central Huainan. Current subsidence monitoring is carried out via conventional methods (e.g. surveying, GPS monuments, aerial photogrammetric survey, etc.). These methods are time-consuming, costly, limited in areal coverage, and produce questionable results. In addition, monitoring/control points are not usually dense enough to assist in fundamentally understanding the mechanisms involved in ground subsidence. So a PS-InSAR based surface subsidence measurement is carrying out in this area.
According to its geographic, geologic background and land use classification results, the surface of Huainan coal mining area contains both rural areas with crops and urban areas with densely man-made structures. So we adopt an integrated method to measure surface subsidence based on PS technique (Fig. 5). Firstly, we group initial SLC datasets into two parts by mask and crop: rural areas and urban areas, and then subdivided SLC datasets in rural area group into two subgroups by seasons based on acquisition time. Secondly, DInSAR processing is carried out in each group and got stacks of differential interferograms. Thirdly, DePSI and StaMPS methods are adopted in urban area and non-urban area respectively. Finally, PS-InSAR derived deformation values in each group are imported into arcGIS for data integration and spatial analysis, so that the deformation map of the whole research area are produced.

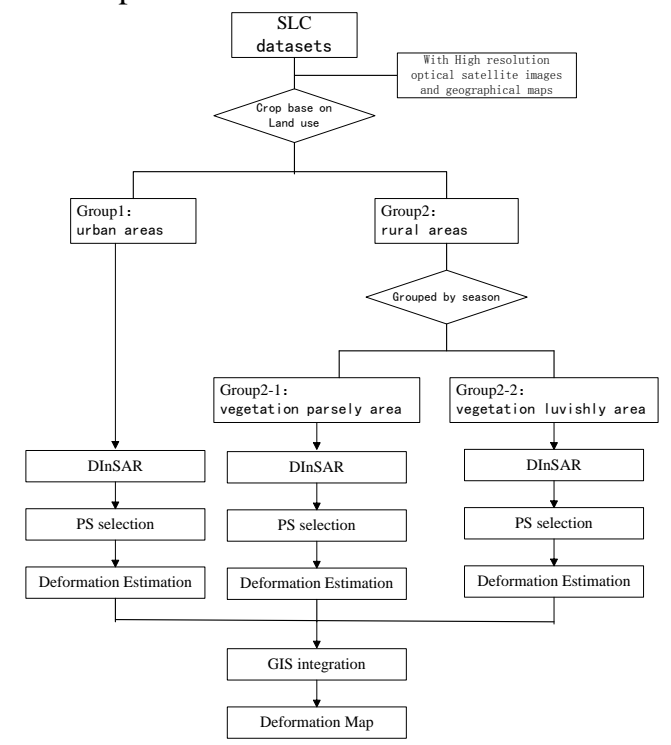

Fig. 3. Flowchart of PS-DInSAR processing in Huainan Coal mining area

\section{CONCLUSIONS}

The PS technique is regarded as an extension of the conventional differential interferometric technique, which demonstrates that using a large number of images is a way to reduce atmospheric artifacts and to obtain highly precise estimation of despite decorrelation. This technique is more efficient and less expensive, compared to conventional groundbased survey methods, therefore it is an operational technique for long-time subtle surface subsidence monitoring and measurement.

\section{ACKNOWLEDGMENT}

This research is supported by National Natural Science Foundation of China (No. 40802080), the National Key Technology R\&D Program (No. 2012BAC10B02) and Huainan Mining Group (No. HNKY-JT-JS-(2011)).

Authors would like to appreciate European Space Agency (ESA) for providing ASAR SLC images under No. 9389. 


\section{REFERENCES}

[1] A. Ferretti, C. Prati, F. Rocca, "Permanent scatterers in SAR interferometry," In: IEEE Proceedings on Geoscience and Remote Sensing Symposium, IGARSS'99, pp. 1528-1530, 1999

[2] A. Perretti, C. Prati, F. Rocca, "Nonlinear subsidence rate estimation using permanent scatterers in differential SAR interferometry," IEEE transactions on geoscience and remote sensing, vol. 38, pp. 2202-2212, 2000.

[3] A. Perretti, C. Prati, F. Rocca, "Permanent scatterers in SAR interferometry," IEEE Transactions on Geoscicence and Remote sensing, vol. 39, pp. 8-20, 2001.

[4] J. J. Sousa, A. M. Ruiz, R. F. Hanssen, L. Bastos, A. J. Gil, J. Galindo-Zaldivar, C. S. Galdeano, "PS-InSAR processing methodologies in the detection of field surface deformationStudy of the Granada basin (Central Betic Cordilleras, southern Spain),". Journal of Geodynamics, vol. 49, pp. 181-189, 2010.

[5] P. Damoah-Afari, "Detecting ground settlement of megacities using InSAR techniques," doctorate thesis, The Hong Kong Polytechnic University, 2008.

[6] V. B. H. Ketelaar, Satellite radar interferometry: subsidence monitoring techniques, Springer, 2009.

[7] C. Prati, A. Ferretti, D. Perissin, "Recent advances on surface ground deformation measurement by means of repeated spaceborne SAR observations," Journal of Geodynamics, vol. 49, pp. 161-170, 2010.

[8] B M. Kampes, Radar interferometry: persistent scaterer technique. Springer, 2006.
[9] M. Lazecky, E. Jirankova, D. Bohmova, 2010. "Usage of InSAR techniques to detect and monitor terrain subsidence due to mining activities,". GeoScience Engineering, vol. LVI, pp. 3249, 2010.

[10] A. J. Hooper, "Persistent scatterer radar interferometry for crustral deformation studies and modeling of volcanic deformation," doctorate thesis, Standford University, 2006.

[11] J. J. Sousa, A. J. Hooper, R. F. Hanssen, L. C. Bastos, A. M. Ruiz, "Persistent scatterer InSAR: a comparision of methodologies based on a model of temporal deformation vs. spatial correlation selection criteria," Remote Sensing of Environment, vol. 115, pp. 2652-2663, 2011.

[12] H. A. Zebker, J. Villasenor, "Decorrelation in interferometric radar echoes," IEEE transactions on geoscience and remote sensing, vol. 30, pp. 950-959, 1992.

[13] Gamma Corporation, "GAMMA Interferometric Point Target Analysis Software (IPTA): Users Guide," unpublished

[14] G. Herrera, R. Tomas, J. M. Lopez-Sanchez, J. Delgado, J.J. Mallorqui, S. Duque, J. Mulas, "Advanced DInSAR analysis on mining areas: La Union case study (Murcia, SE Spain)," Engineering geology, vol. 90, 148-159, 2007.

[15] H. C. Jung, S. W. Kim, H. S. Jung, K. D. Min, J. S. Won, "Satellite observation of coal mining subsidence by persistent scatterer analysis," Engineering Geology, vol. 92, pp. 1-13, 2007. 